\title{
Acute medical units during the first wave of the COVID-19 pandemic: a cross-national exploratory study of impact and responses
}

\author{
Authors: John TY Soong, ${ }^{\mathrm{A}}$ Audrey LA Wong, ${ }^{\mathrm{B}}$ Imogen O'Connor, ${ }^{\mathrm{C}}$ Milka Marinova, ${ }^{\mathrm{D}}$ Dale Fisher ${ }^{\mathrm{E}}$ and Derek BellF
}

\section{Background}

The COVID-19 pandemic represents one of the greatest ever challenges for healthcare. In the UK and beyond, acute medical units (AMUs) are the first point of assessment and care for the majority of medical inpatients. By their design and systems, they inevitably played an important role in the COVID-19 response but to date little has been published on how the COVID-19 pandemic has affected how AMUs have reorganised their resources, processes and structure.

\section{Methods}

This retrospective study in August 2020 of 10 AMUs across Europe and Australasia used a standardised questionnaire to investigate existing practice and structure of AMUs, the national context of local hospital experience, changes to practice during the COVID-19 pandemic and views regarding future practice.

\section{Results}

Changes to AMU structure, process and organisation are described in two contexts: preventing and controlling the spread of COVID-19 and adding value to the patient's acute care journey in the local context. We describe novel practices that have arisen and highlight areas of concern.

\section{Conclusions}

The AMUs were able to adapt to meet the demands of acute care delivery during the first wave of the COVID-19 pandemic. Operational planning and prioritisation of resources must be optimised to ensure sustainability of these services for future waves.

Authors: Aconsultant, National University Hospital, Singapore, assistant professor, National University of Singapore, Singapore, and honorary senior clinical lecturer, Imperial College London, London, UK; Bsenior consultant, National University Hospital, Singapore, and assistant professor, National University of Singapore, Singapore; research assistant, Imperial College London, London, UK, and research assistant, Applied Research Collaboration Northwest London, UK; ${ }^{\mathrm{D}}$ Clinical research fellow, Imperial College London, London, UK; Esenior consultant, National University Hospital, Singapore, and professor of medicine, National University of Singapore, Singapore; Fprofessor of acute medicine, Imperial College London, London, UK
KEYWORDS: AMU, COVID-19, structures, processes, response

DOI: $10.7861 /$ clinmed.2021-0150

\section{Introduction}

The COVID-19 pandemic represents one of the greatest ever challenges for healthcare. In the UK and beyond, acute medical units (AMUs) are the first point of assessment and care for the majority of medical inpatients, and there is evidence that they can reduce length of hospital stay ${ }^{2}$ and mortality ${ }^{3}$ and improve patient flow from emergency departments to medical beds ${ }^{4}$ without increase in readmissions. ${ }^{5}$ By their design, they have inevitably played an important role in the COVID-19 response but to date little has been published on how the COVID-19 pandemic has affected the function of $\mathrm{AMUs}^{6}$ and how they reorganised their resources, processes and structures to meet the challenges of this pandemic.

This retrospective cross-national study explores this response: how structures and processes have been adapted in order to continue to add value to the acute care patient journey and what innovative practices have emerged. The impact of infection prevention and control measures on AMU design, staffing and function is also addressed, as well as the impact on education, audit and research. Here, we share international experiences from 10 AMUs after experiencing the first wave of the pandemic in their respective regions.

\section{Methods}

\section{Study questionnaire}

A questionnaire (see supplementary material S1) was iteratively developed (by the authors, with field testing by colleagues across three AMUs internationally) to encompass the existing practice and structure of AMUs, the national context of local hospital experience, changes to practice during the COVID-19 pandemic and views regarding future practice.? The study was undertaken in August 2020.

\section{Sampling}

The authors identified potential recruitment sites from members of the Society for Acute Medicine. A range of hospitals were chosen to cover geographical breadth (UK, Europe, Australia, Asia), 
hospital category (tertiary academic centres and district general hospitals) and AMU (sub)type (ie case mix and whether the unit provided ambulatory care or was a frailty unit). ${ }^{8}$ Of the 15 units approached, 10 units agreed to participate. These comprised a mix of secondary and tertiary acute care centres.

\section{Medium}

The clinical leads from each AMU were invited to participate via an online questionnaire designed on the Qualtrics ${ }^{\circledR}$ platform. A single reminder was sent after 4 weeks. Where there was ambiguity in specific responses, the participating site was contacted directly for clarification.

\section{Results}

\section{Admission volume}

10 hospitals from the UK (England and Scotland), Australia, Malaysia, the Netherlands and Singapore participated. The hospitals reported the first major epidemiologic peak of COVID-19 activity from March to June 2020. The first peak months for COVID-19 activity varied even for hospitals in the same country but centered around each country's peak. These peaks were associated with decreased total volume of patients admitted to the AMUs (range 20-60\%). Fig 1 displays the peak months of the first wave of COVID-19 activity.

\section{Case mix}

The participating AMUs report some variation in their routine case mix, reflecting different objectives or roles for the unit during the pandemic. Table 1 describes change to the case mix in AMUs before and during the COVID-19 pandemic. (The full inclusion and exclusion criteria for the AMUs before and during the COVID pandemic are given in the expanded version of Table 1 in supplementary material S2.) During the first wave of the COVID-19 pandemic, six AMUs excluded COVID-19-positive and suspect patients, three units created COVID-19 beds and operated on a mixed model, and one unit admitted exclusively COVID-19 positive and suspect patients, consequently ceasing all AMU functions. Of the three units that operated a mixed model, one unit $(\mathrm{RH})$ repurposed and managed 10 additional beds on a surgical ward as a COVID-19 area; one unit (UHND) converted 12 existing beds within the AMU into an acute respiratory unit for COVID-19 patients; and one unit (FVH) created a separate red zone for suspected or proven COVID-19 and yellow zone for low-likelihood patients. NUH Singapore, which operated a non-COVID-19 model, extended the threshold for transfer to medical wards from 72 hours to 7 days.

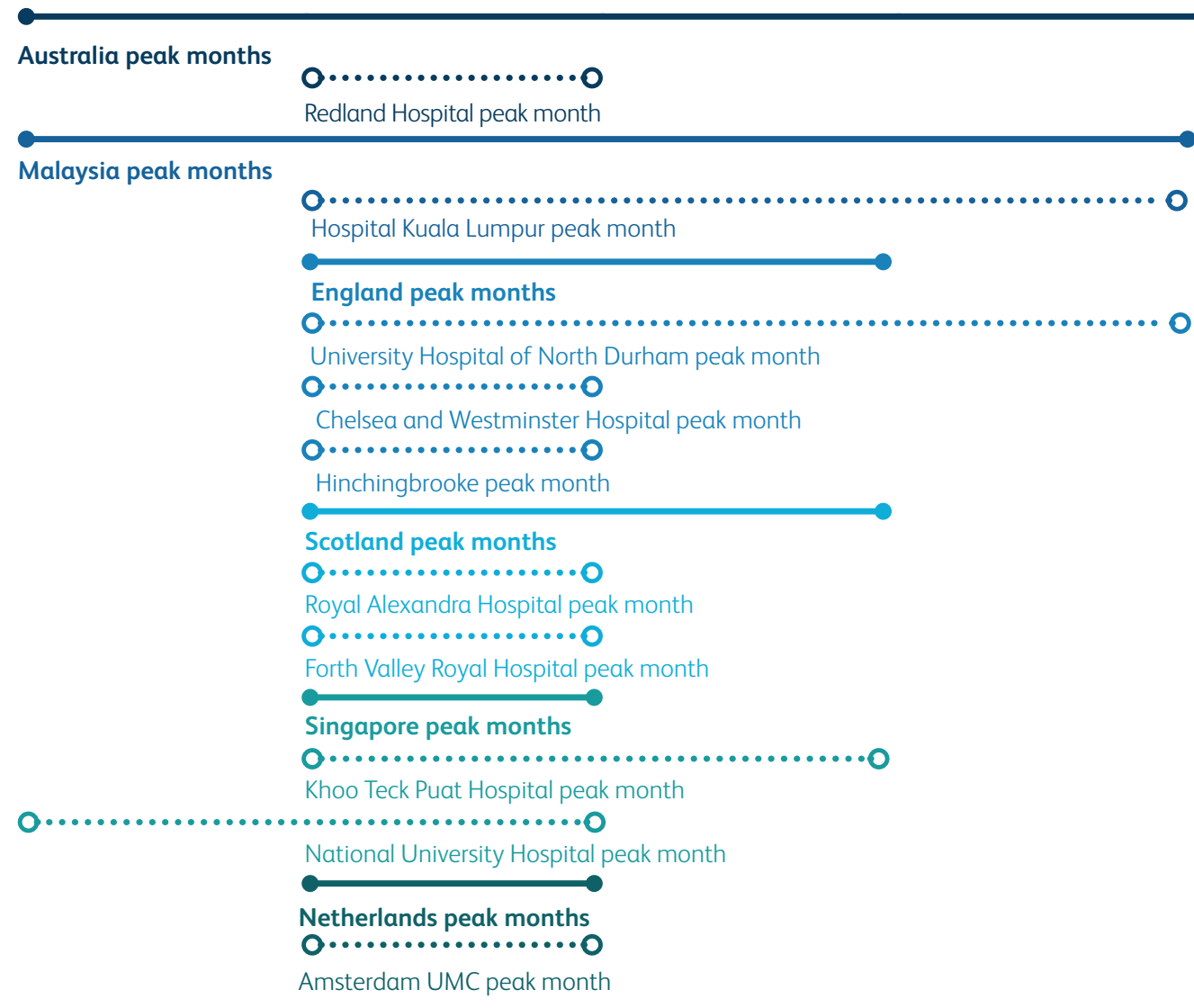

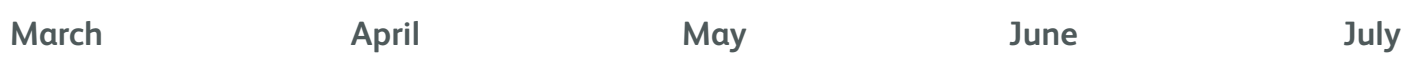

Fig 1. Peak months for country and hospitals for COVID-19 pandemic. 
Table 1. Case mix in acute medical units before and during the COVID-19 pandemic

\begin{tabular}{lllll} 
Hospital & City, country & Acute care services & $\begin{array}{l}\text { Case mix during } \\
\text { COVID-19 pandemic }\end{array}$ & $\begin{array}{c}\text { Inclusion and } \\
\text { exclusion criteria }\end{array}$ \\
Redland Hospital & $\begin{array}{l}\text { Cleveland, Redland City, } \\
\text { Australia }\end{array}$ & Secondary & Mixed (separate ward) & No change \\
$\begin{array}{l}\text { University Hospital of North } \\
\text { Durham } \\
\text { Chelsea and Westminster }\end{array}$ & $\begin{array}{l}\text { Durham, England } \\
\text { Hospital }\end{array}$ & Secondary & Mixed & Unavailable \\
$\begin{array}{l}\text { Hinchingbrooke } \\
\text { Hospital Kuala Lumpur }\end{array}$ & Huntingdon, England & Secondary/tertiary & Non-COVID & NIV/CPAP halted \\
$\begin{array}{l}\text { Amsterdam UMC } \\
\text { Royal Alexandra Hospital }\end{array}$ & Amsterdam, Netherlands & Tertiary & Non-COVID & No change \\
Forth Valley Royal Hospital & Larbert, Scotland & Non-COVID & No change \\
Khoo Teck Puat Hospital & Singapore, Singapore & Secondary & Non-COVID & Unavailable \\
National University Hospital & Singapore, Singapore & Tertiary & Non-COVID & No change \\
& & Secondary & Mixed & No change \\
\hline
\end{tabular}

MRSA = methicillin-resistant Staphylococcus aureus; NIV/CPAP = non-invasive ventilation / continuous positive airway pressure.

\section{Transmission control and contact tracing}

Nine of the participating units felt that healthcare staff had access to sufficient personal protective equipment (PPE) during their respective peak periods. A Scottish unit felt they had insufficient mask type available and conflicting guidance on cardiopulmonary resuscitation. Nine of the participating hospitals had an internal contact tracing policy for patients who tested positive in an area of the hospital not designated to receive COVID-19 patients. These included isolating the patient (eg pulmonary unit) and contact tracing (by infection control team, epidemiology unit, infectious diseases team or via state-level mechanisms). One English unit felt their contact tracing policy was not sufficiently robust.

Nine of the participating hospitals had an internal contact tracing policy for staff members who tested positive for COVID-19 (by infection control team, occupational health team, epidemiology unit or government initiatives). Isolation policies for staff members varied by testing capacity. For countries which lacked testing capacity, either staff were asked to stay home for 14 days after exposure or from onset of symptoms, or testing was restricted to those who developed symptoms. Only six of the hospitals had a policy to separate consultant staff working with both COVID-19-positive/suspect patients and non-COVID-19 patients (for five hospitals, the wash-out period was less than 24 hours, and for one it was 2-7 days). Seven of the hospitals had a policy to separate junior medical staff working with both COVID19-positive/suspect and non-COVID-19 patients (for six hospitals, the wash-out period was less than 24 hours and for one it was 2-7 days). None of the participating AMUs instituted routine staff screening for COVID-19 with a viral swab. The three English units instituted routine screening for COVID-19 antibodies in the months of April, May and June respectively.

Seven AMUs underwent infrastructural changes. Four units (CWH, KTPH, HKL, UHND) erected temporary fixtures (plexiglass or other solid partitions). Three units (FVRH, HH, RAH) made changes to the waiting area (reduced capacity or removed it completely and restricted to patients only). Four units (FVRH, HH, HKL,
UHND) reduced bed capacity to accommodate infection control distancing. The Singapore unit (KTPH) that became a dedicated COVID-19 ward changed staff flow to minimise spread (including providing dedicated staff areas eg pantry and showers). Another Singapore unit (NUH) segregated staff who worked on COVID-19 areas versus non-COVID-19 areas (eg arranging staggered mealtimes). Two units (HKL, RA) instituted triage systems for acute respiratory illness.

\section{Current and forward operational practices}

Work practices that had halted or decreased significantly due to the COVID-19 pandemic included bedside teaching, team socialising, quality improvement projects and ambulatory care services. All the units planned to return to these practices once the pandemic had resolved. Work practices which evolved during the pandemic that participating units hoped to cease include increased distancing between patients, increased frequency of on-calls and AMU (or part of AMU) functioning as an acute respiratory unit (two units). Novel work practices that units wish to continue include increased use of telemedicine for clinical and educational purposes (including virtual clinics, ambulatory care pathways and remote meetings/teaching).

This questionnaire was conducted at the end of the first wave for most of the participating hospitals. At point of study, the three English and single Malaysian units would in future screen all patients for admission to the AMU, whereas the others would screen only high-risk (definitions varied by country) or symptomatic patients. All units had returned to baseline admission levels prior to the COVID-19 pandemic, with the exception of one unit in Singapore, which remained a COVID-19 admission ward.

When participating units were asked about preparation for the next wave, several themes emerged. Two units described all COVID-19 suspected patients being routed to a dedicated COVID-19 hospital, rather than to any hospital. ${ }^{9}$ There was agreement that point-of-care testing for COVID-19 would be a priority. It was noted 
Table 2. Acute medical unit structure changes during the COVID-19 pandemic

\begin{tabular}{|c|c|c|c|c|}
\hline Hospital & Before/during COVID & Ambulatory care unit & Acute frailty unit & Other \\
\hline \multirow[t]{2}{*}{ Redland Hospital } & Before & $\checkmark$ & $\boldsymbol{x}$ & 5 HDU beds \\
\hline & During & $\sim$ & $\boldsymbol{x}$ & $\sim$ \\
\hline \multirow[t]{2}{*}{ University Hospital of North Durham } & Before & $\checkmark$ & $\mathbf{x}$ & $\boldsymbol{x}$ \\
\hline & During & $\downarrow$ & $\boldsymbol{x}$ & $\boldsymbol{x}$ \\
\hline \multirow[t]{2}{*}{ Chelsea and Westminster Hospital } & Before & $\checkmark$ & $\checkmark$ & 9 HDU beds \\
\hline & During & $\downarrow$ & $\boldsymbol{x}$ & $\sim$ \\
\hline \multirow{2}{*}{ Hinchingbrooke Hospital } & Before & $\checkmark$ & $\boldsymbol{x}$ & Seated treatment area \\
\hline & During & $\uparrow$ & $\boldsymbol{*}$ & $\sim$ \\
\hline \multirow[t]{2}{*}{ Hospital Kuala Lumpur } & Before & $\checkmark$ & $\boldsymbol{x}$ & $\boldsymbol{x}$ \\
\hline & During & $\sim$ & $\boldsymbol{x}$ & $\boldsymbol{x}$ \\
\hline \multirow[t]{2}{*}{ Amsterdam UMC } & Before & $\boldsymbol{x}$ & $\boldsymbol{x}$ & $\boldsymbol{x}$ \\
\hline & During & $\boldsymbol{x}$ & $\boldsymbol{x}$ & $\boldsymbol{*}$ \\
\hline \multirow[t]{2}{*}{ Royal Alexandra Hospital } & Before & $\checkmark$ & $\checkmark$ & $\boldsymbol{x}$ \\
\hline & During & $\sim$ & $\boldsymbol{x}$ & $*$ \\
\hline \multirow[t]{2}{*}{ Forth Valley Royal Hospital } & Before & $\checkmark$ & $\boldsymbol{x}$ & $\boldsymbol{x}$ \\
\hline & During & $\sim$ & $\boldsymbol{x}$ & $\mathbf{x}$ \\
\hline \multirow[t]{2}{*}{ Khoo Teck Puat Hospital } & Before & $\checkmark$ & $\checkmark$ & $\boldsymbol{x}$ \\
\hline & During & $\boldsymbol{x}$ & $\boldsymbol{x}$ & $\boldsymbol{x}$ \\
\hline \multirow[t]{2}{*}{ National University Hospital } & Before & $\boldsymbol{x}$ & $\boldsymbol{x}$ & $\boldsymbol{x}$ \\
\hline & During & $\boldsymbol{x}$ & $\boldsymbol{x}$ & $\boldsymbol{x}$ \\
\hline
\end{tabular}

that non-COVID-19 activity was still a large proportion of daily work, and business continuity measures to ensure sustainability of these services remain important. One English unit perceived PPE and equipment stock levels as a crucial priority, coupled with improved rota management and sustainable staff separation.

\section{Auxiliary acute services}

Table 2 describes additional changes to AMUs and auxiliary services based on local health need.

\section{Ambulatory care}

Eight of the 10 units had existing ambulatory care services; of these, four (FVH, RA, RH, HKL) maintained the same number of beds/trolleys/chairs, one $(\mathrm{HH})$ increased capacity, two decreased capacity (CWH, UHND) and one closed (KTPH). In terms of activity, six out of eight ambulatory care units described decreased activity, while two units described increased activity. Ambulatory care services were impacted by COVID-19 in various ways. The participating unit from Australia managed stable COVID-19 patients $(n=10)$ via a hospital-at-home service. One unit $(\mathrm{RAH})$ instituted separate pathways for COVID-19-positive and -negative patients, and another (UHND) moved their ambulatory services to a different location. Full changes to ambulatory care capacity are described in supplementary material S2.

Three ambulatory units ( $\mathrm{CWH}, \mathrm{RAH}, \mathrm{HKL}$ ) reduced the number of beds and trolleys to increase the spacing between beds to 1 metre.
One ambulatory unit $(\mathrm{HH})$ removed beds and replaced these with distanced trolleys and chairs to be able to continue services.

High-dependency care

Two of the 10 units had existing critical care services (high dependency units [HDUs] or Level 1 beds) and these were retained during the pandemic ( $\mathrm{RH}$ had five HDU beds; $\mathrm{CWH}$ had nine Level 1 beds). These sites provided non-invasive ventilation (NIV) during the pandemic for COVID-19 negative patients. UHND introduced a respiratory unit and provided NIV for COVID-19 positive patients. $\mathrm{CWH}$ increased their number of NIV machines.

\section{Frailty unit}

Three of the 10 units (CWH, KTPH and RAH) had acute frailty units and all ceased functions due to staff shortages.

\section{Telemedicine}

Eight participating hospitals describe using telemedicine services in the AMU and ambulatory care settings, of which five were new services in response to COVID-19.

\section{Resources and data acquisition}

Table 3 (expanded version given in supplementary material S2) displays changes in AMU bed capacity and consultant staffing before and during the COVID-19 pandemic. Five out of 10 AMUs 
Table 3. Acute medical unit bed capacity and consultant staffing before and during COVID-19 pandemic

\begin{tabular}{|c|c|c|c|c|c|}
\hline Hospital & $\begin{array}{l}\text { Before/during } \\
\text { COVID }\end{array}$ & $\begin{array}{l}\text { Number of } \\
\text { hospital beds }\end{array}$ & $\begin{array}{l}\text { AMU beds } \\
\mathrm{N}(\%)\end{array}$ & $\begin{array}{l}\text { Ratio of AMU to } \\
\text { hospital beds }\end{array}$ & $\begin{array}{l}\text { Consultants per month } \\
\text { on clinical roster for AMU }\end{array}$ \\
\hline \multirow[t]{2}{*}{ Redland Hospital } & Before & 172 & $48(28 \%)$ & 1 to 4 & 6 \\
\hline & During & & $58(34 \%)$ & 1 to 3 & 7 \\
\hline \multirow[t]{2}{*}{ University Hospital of North Durham } & Before & 523 & $48(9 \%)$ & 1 to 11 & 16 \\
\hline & During & & $36(7 \%)$ & 1 to 15 & 24 \\
\hline \multirow[t]{2}{*}{ Chelsea and Westminster Hospital } & Before & 505 & $54(11 \%)$ & 1 to 9 & 17 \\
\hline & During & & $54(11 \%)$ & 1 to 9 & 7 \\
\hline \multirow[t]{2}{*}{ Hinchingbrooke Hospital } & Before & 220 & $15(7 \%)$ & 1 to 15 & 15 \\
\hline & During & & $9(4 \%)$ & 1 to 24 & 15 \\
\hline \multirow[t]{2}{*}{ Hospital Kuala Lumpur } & Before & 2100 & $24(1 \%)$ & 1 to 88 & 4 \\
\hline & During & & $20(1 \%)$ & 1 to 105 & 4 \\
\hline \multirow[t]{2}{*}{ Amsterdam UMC } & Before & 1002 & $24(2 \%)$ & 1 to 42 & 8 \\
\hline & During & & $24(2 \%)$ & 1 to 42 & 10 \\
\hline \multirow[t]{2}{*}{ Royal Alexandra Hospital } & Before & 618 & $30(5 \%)$ & 1 to 21 & 31 \\
\hline & During & & $60(10 \%)$ & 1 to 10 & 24 \\
\hline \multirow[t]{2}{*}{ Forth Valley Royal Hospital } & Before & 500 & $70(14 \%)$ & 1 to 7 & 24 \\
\hline & During & & $44(9 \%)$ & 1 to 11 & 21 \\
\hline \multirow[t]{2}{*}{ Khoo Teck Puat Hospital } & Before & 590 & $33(6 \%)$ & 1 to 18 & 3 \\
\hline & During & & $0(0 \%)$ & 0 to 590 & 0 \\
\hline \multirow[t]{2}{*}{ National University Hospital } & Before & 1239 & $36(3 \%)$ & 1 to 34 & 8 \\
\hline & During & & $36(3 \%)$ & 1 to 34 & 8 \\
\hline
\end{tabular}

(UHND, HH, HKL, FVRH, KTPH) reduced bed capacity as part of infection control measures during the pandemic. Three hospitals (CWH, AUMC, NUH) had no change in capacity and two hospitals increased capacity. $\mathrm{RH}$ repurposed 10 beds on an adjacent surgical ward to manage COVID-19 patients and RAH opened a dormant ward adjacent to the existing AMU to manage non-COVID-19 patients.

In response to COVID-19, three AMUs (RH, UHND, AUMC [two of which operated a mixed model during the pandemic]) increased the number of consultants rostered for AMU, three hospitals $(\mathrm{HH}, \mathrm{HKL}, \mathrm{NUH})$ had no change in consultant coverage and four AMUs (CWH, RAH, FVRH, KTPH [one of which operated a mixed model during the pandemic]) decreased consultant coverage. The breakdown of generalist, specialist and acute physician consultant cover for AMUs are described in supplemental material S2.

It was noted that operational system and patient flow data were not readily available. Only three participating units were able to provide scant data for mean length of stay on AMU, 28/30-day emergency readmission, inpatient mortality and pre-mid-day discharge rate for the hospital by the time the online study closed. These data have therefore been excluded from analysis.

\section{Research, audit and education}

Four of the AMUs participated in clinical studies during the pandemic. This included drug trials for COVID-19-positive patients as well as observational studies of recovery and risk prediction tools using point-of-care ultrasound and chest CT. No units undertook clinical audits during the pandemic. Seven units continued to provide postgraduate medical education, though this was more limited. Five units continued bedside teaching, by utilising telemedicine platforms, safe distancing measures and PPE. Only one unit in Scotland continued allowing undergraduate teaching on the AMU. Four units from the UK and the Netherlands unit recruited final year medical students to support clinical services.

\section{Discussion}

We report the experience and practice of $10 \mathrm{AMUs}$ worldwide as they responded to meet the challenges of the COVID-19 pandemic. Although heterogenous in terms of patient case mix and unit design to meet local needs, the resultant requirements to triage and isolate patients significantly affected operations, patient flow, service provision and consequently staffing levels.

New information about the SARS-CoV-2 virus and potential beneficial treatments are rapidly emerging. Guidelines for treatment of COVID-19 are in evolution as trial evidence develops. ${ }^{10}$ However, healthcare organisations require necessary changes to operational

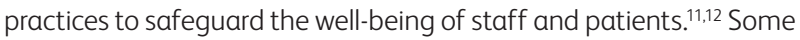
potential learning points can be gleaned from the results of this study. 


\section{Functional adaptation}

AMUs have the ability to adapt and flex their function to meet the demands of the hospital and local health system. In our study, we observe that for units whose primary purpose shifted to treat

COVID-19-negative patients, their practices and processes altered to better accommodate patient flow and continuity (eg extended length of stay over 72 hours, ambulatory care services,

telemedicine). Some AMUs, particularly those whose original function was to treat high acuity patients, converted rapidly and effectively into a treatment ward for COVID-19 positive patients. Earlier surveys of AMUs in UK by the Society for Acute Medicine suggest many units are managing patients with COVID suspect or positive patients, and that activity was high at peak. ${ }^{13}$ In response to future outbreaks, we recommend that AMUs proactively define their role and alter processes to best add value to patients within the AMU as well as to the hospital as a whole, given contextual challenges. Particular attention should be paid to the use of physical space, as they will need to maintain appropriate distancing between beds, trolleys and chairs in wards and waiting areas, as well as clearly defined flow paths for staff and patients to avoid cross contamination. Though three units operated a mixed model, we offer a word of caution. The complexities of maintaining appropriate social distancing between beds $(>1.0 \mathrm{~m}),{ }^{14}$ separate air filtration systems and isolating flow of patients, staff and relatives may make this untenable in the medium to long term without more permanent solutions to space, staff protection and patient flow. Of note, two of the units that operated a mixed model had concerns about staff protection and contact tracing.

\section{Human resource sustainability}

Our study suggests that in the hurry to meet the demands of a rapidly escalating service, business continuity practices may be suboptimal. Though it may not be possible (or even desirable) to have staff dedicated to purely COVID-19/non-COVID-19 clinical services, it is imperative that there is a 'wash-out' period between exposure to COVID-19 positive or suspect patients and COVID-19 negative patients. Ideally this period should be 14 days, though 7 days should be the minimum..$^{15}$ There have been reports of nosocomial spread, ${ }^{16,17}$ which may be preventable with proactive planning and preparedness. ${ }^{18,19}$ This is particularly important as the non-COVID-19 clinical burden remained significant ${ }^{6}$ throughout the pandemic and is likely to increase. Equally, appropriate measures and monitoring systems are needed to maintain staff wellbeing for sustainability of service. ${ }^{20}$ The impact of vaccination on the ability to maintain safe clinical areas will require further study.

\section{Routine testing}

More controversially, regular and routine testing of healthcare staff may be appropriate. A recent Scottish study suggested that one in six hospital admissions for COVID-19 were healthcare staff, and their household members had up to three times increased risk of COVID-19 related hospital admission. ${ }^{21}$ Another study suggests that asymptomatic viral carriage in frontline healthcare workers (including housekeeping) to be as high as $34.5 \%{ }^{22}$ One of the sites had policies for staff surveillance (for symptoms) and assessment (with testing where appropriate). ${ }^{23}$ At the time of writing, there was a pilot in some UK sites where frontline staff were being tested twice a week. Regular and routine testing for COVID-19 should be considered if the population likelihood of COVID-19 is high (eg nursing homes), or if the consequences of a cluster spread is high (eg healthcare workers). For sustainability, once capacity of testing is sufficient, we would recommend routine staff testing for COVID-19. At the time of writing, two COVID-19 vaccines were approved for use, with the majority of countries prioritising frontline healthcare workers for vaccination.

\section{Telecommunication technology}

Our study demonstrates that there are both opportunities and a willingness to increase the use and role of telecommunication technology for many traditionally in-person activities, from education (eg virtual grand rounds) to operations (meetings between staff caring for COVID-19-positive and -negative patients) and clinical function (telemedicine for ambulatory emergency care and discharge follow-ups). ${ }^{24} \mathrm{~A}$ recent study reviewed rapid remote specialty consults by liaison neurology as a means of improving speed and reach of services. ${ }^{25}$ The COVID-19 pandemic has driven the rapid evolution and uptake of telemedicine, and studies of effectiveness and impact should be a priority. ${ }^{26}$

\section{Transmission control policy}

Our study encouragingly finds that the majority of AMUs had clear policies for patients and staff who were exposed to COVID-19, which included contact tracing and isolation. These policies did vary according to resources available and context. Most units felt they had sufficient PPE supplies during their respective peaks. This contrasts with a recent national study in Japan, where shortage of PPE and unclear isolation criteria were of concern to practising acute physicians..$^{15} \mathrm{~A}$ Scottish unit in our study described a lack of clarity in national guidelines for what constituted optimal PPE for different clinical scenarios. We recommend that standardised operational policies are disseminated clearly to all healthcare providers.

\section{Operational data}

It was concerning that very few units had access to readily available, high-quality basic operational data with regards to patient flow and outcomes. Acute care systems need to be able to regularly monitor basic indices to ensure that they have operational intelligence to be responsive to surges in patient need, but also to ensure no harm is being done with changes in processes. ${ }^{9}$ We recommend that 7- and 30 -day readmission rates, length of hospital stay, discharge before mid-day rates (or measure of delayed discharge), inpatient mortality rates and nosocomial infection rates should be included in a minimum dataset of what should be tracked on a regular basis, combined with measures of patient experience. Routine data collection and analysis must become a priority.

\section{Non-invasive ventilation}

Evidence for the use of NIV for COVID-19-positive patients is evolving. ${ }^{27}$ Early intubation and mechanical ventilation are initially preferred for COVID-19 patients with severe respiratory failure. Emerging experience report that, alongside high-flow nasal oxygen, NIV may be used as a bridge or even prevent the 
need for mechanical ventilation, though this viewpoint remains contentious. ${ }^{28}$ Given risks of spread, strict infection control protocols with appropriate isolation rooms, and 'washout' periods for staff who work in these areas are crucial.

\section{Limitations}

This online study is not a systematic survey representative of all AMUs, but covers a selection of units within different countries, hospital type and with different acute services. This allowed a wide range of experience and practices to be reflected. The majority of AMUs could not produce data pertaining to patient flow and outcomes. It is not possible from these findings to propose international benchmarks. Based on the results, we describe novel practices that have arisen and make suggestions for further research and systematic surveys.

\section{Conclusion}

The AMUs studied were able to flex and adapt to meet the demands of acute care delivery during the first wave of the COVID-19 pandemic. Operational planning and prioritisation of resources must be optimised to ensure sustainability of these services for future waves.

\section{Summary}

\section{What is known?}

AMUs, by design, have played a crucial role in acute medical care during the COVID pandemic. Little has been published on how the pandemic has affected the functioning of AMUs.

\section{What is the question?}

How has the COVID-19 pandemic affected the function of AMUs? In particular, how have they reorganised their resources, processes and structures to meet the challenges of this pandemic?

\section{What is found?}

The AMUs studied have flexed and adapted to meet the demands of acute care delivery during the first wave of the COVID-19 pandemic in diverse ways. These are described.

\section{What is the implication for practice now?}

Operational planning and prioritisation of resources must be optimised to ensure sustainability of AMU services for future pandemic waves.

\section{Supplementary material}

Additional supplementary material may be found in the online version of this article at www.rcpjournals.org/clinmedicine: S1 - Online questionnaire for AMU practice before and during the COVID-19 pandemic.

S2 - Extended versions of Table 1, Table 2 and Table 3.

\section{References}

1 Cooksley T. Editorial - COVID-19: Exemplifying the importance and challenges of acute medicine. Acute Med 2020;19:174-5.

2 Reid LEM, Dinesen LC, Jones MC, et al. The effectiveness and variation of acute medical units: a systematic review. Int J Qual Health Care 2016;28:433-46.
3 Conway R, Byrne D, Cournane S, O'Riordan D, Silke B. Fifteenyear outcomes of an acute medical admission unit. Ir J Med Sci 2018:187:1097-105.

4 Scott I, Vaughan L, Bell D. Effectiveness of acute medical units in hospitals: a systematic review. Int J Qual Health Care 2009;21:397-407.

5 St Noble V], Davies G, Bell D. Improving continuity of care in an acute medical unit: initial outcomes. QJM 2008;101:529-33.

6 Lange PW, Gazzard M, Walker S et al. Where are our patients? Retrospective cohort study of acute medical unit admissions during and prior to the COVID-19 pandemic. Int Med J 2020;50:1132-4.

7 Kelley K, Clark B, Brown V, Sitzia J. Good practice in the conduct and reporting of survey research. Int J Qual Health Care 2003:15:261-6.

8 Ponto J. Understanding and evaluating survey research. J Adv Pract Oncol 2015;6:168-71.

9 Oda J, Takashi M, Matsuyama S et al. JAAM Nationwide Survey on the response to the first wave of COVID-19 in Japan Part II: How the medical institutions overcame the first wave and how to prepare in future? Acute Med Surg 2020;7:e592.

10 Bhimraj A, Morgan RL, Shumaker AH et al. COVID-19 Guideline, Part 1: Treatment and management. Infectious Diseases Society of America, 2020. www.idsociety.org/practice-guideline/covid-19guideline-treatment-and-management/.

11 International Labour Organization. COVID-19 and health facilities: Checklist of measures to be taken in health facilities. ILO, 2020. Available from: www.ilo.org/sector/Resources/publications/ WCMS_750808/lang--en/index.htm [Accessed 27 December 2020].

12 World Health Organization. Rapid hospital readiness checklist: Interim Guidance. WHO, 2020. Available from www.who.int/ publications-detail-redirect/WHO-2019-nCoV-hospital-readinesschecklist-2020.1 [Accessed 27 December 2020].

13 Scriven N. Hospital winter planning must include regular Covid-19 testing of all frontline staff. BMJ Opinion, 18 October 2020. https:// blogs.bmj.com/bmj/2020/10/18/nick-scriven-hospital-winter-planning-must-include-regular-covid-19-testing-of-all-frontline-staff/.

14 World Health Organization. Minimum requirements for infection prevention and control (IPC) programmes. WHO, 2019. www.who. int/infection-prevention/publications/min-req-IPC-manual/en/ [Accessed 9 February 2021].

15 Centers for Disease Control and Prevention. Interim infection prevention and control recommendations for healthcare personnel during the coronavirus disease 2019 (COVID-19) pandemic. CDC, 2020. Available from: www.cdc.gov/coronavirus/2019-ncov/hcp/infectioncontrol-recommendations.html [Accessed 27 December 2020].

16 Jewkes SV, Zhang Y, Nicholl DJ. Nosocomial spread of COVID-19: lessons learned from an audit on a stroke/neurology ward in a UK district general hospital. Clin Med 2020;20:e173-7.

17 Rhee C, Baker M, Vaidya $V$ et al. Incidence of nosocomial COVID-19 in patients hospitalized at a large us academic medical center. JAMA Netw Open 2020;3:e2020498.

18 Archuleta S, Cross G, Somani ] et al. Responding to COVID-19: how an academic infectious diseases division mobilized in Singapore. BMC Medicine 2020:18:179.

19 Lum BX, Liu EH, Archuleta S et al. Establishing a new normal for hospital care: a whole of hospital approach to coronavirus disease 2019 (COVID-19). Clin Infect Dis 2020, in press (DOI: 10.1093/cid/ ciaa1722).

20 Oda J, Tanabe S, Nishimura T et al. JAAM Nationwide Survey on the response to the first wave of COVID-19 in Japan. Part I: How to set up a treatment system in each hospital. Acute Med Surg 2020;7:e614.

21 Shah ASV, Wood R, Gribben C et al. Risk of hospital admission with coronavirus disease 2019 in healthcare workers and their households: nationwide linkage cohort study. BMJ 2020;371:m3582.

22 Shields A, Faustini SE, Perez-Toledo M et al. SARS-CoV-2 seroprevalence and asymptomatic viral carriage in healthcare workers: a cross-sectional study. Thorax 2020;75:1089-94. 
23 Hwang J, Yong E, Cheong K et al. Responding to the COVID-19 pandemic: The role of occupational health services in a tertiary hospital in Singapore. J Occup Health 2020;62:e12172.

24 Greenhalgh T, Koh GCH, Car J. Covid-19: a remote assessment in primary care. BMJ 2020;368:m1182.

25 Fuller GN. Improving liaison neurology services. Pract Neurol 2020;20:494-8.

26 Hendy ], Chrysanthaki T, Barlow ] et al. An organisational analysis of the implementation of telecare and telehealth: the whole systems demonstrator. BMC Health Serv Res 2012;12:403.

27 Dobler CC, Murad MH, Wilson ME. Noninvasive positive pressure ventilation in patients with COVID-19. Mayo Clin Proc 2020:95:2594-601.
28 Arulkumaran N, Brealey D, Howell D, Singer M. Use of non-invasive ventilation for patients with COVID-19: a cause for concern? Lancet Resp Med 2020;8:e45.

Address for correspondence: Dr John Tshon Yit Soong, Division of Advanced Internal Medicine, University Medicine Cluster, National University Hospital, 1E Kent Ridge Road, NUHS Tower Block Level 10, Singapore 119228.

Email: John_Soong@nuhs.edu.sg

Twitter: @JSoongNUHS 